\title{
AN ANALYTICAL CHARACTERIZATION FOR AN OPTIMAL CHANGE OF GAUSSIAN MEASURES
}

\author{
HENRY SCHELLHORN
}

Received 25 February 2006; Revised 9 June 2006; Accepted 9 June 2006

We consider two Gaussian measures. In the "initial" measure the state variable is Gaussian, with zero drift and time-varying volatility. In the "target measure" the state variable follows an Ornstein-Uhlenbeck process, with a free set of parameters, namely, the timevarying speed of mean reversion. We look for the speed of mean reversion that minimizes the variance of the Radon-Nikodym derivative of the target measure with respect to the initial measure under a constraint on the time integral of the variance of the state variable in the target measure. We show that the optimal speed of mean reversion follows a Riccati equation. This equation can be solved analytically when the volatility curve takes specific shapes. We discuss an application of this result to simulation, which we presented in an earlier article.

Copyright (C) 2006 Henry Schellhorn. This is an open access article distributed under the Creative Commons Attribution License, which permits unrestricted use, distribution, and reproduction in any medium, provided the original work is properly cited.

\section{Introduction}

We consider two Gaussian measures. In the "initial" measure the state variable is Gaussian, with zero drift, (we chose zero drift for ease of exposition, but the same development applies to a nonzero drift) and time-varying volatility. In the "target measure" the state variable follows an Ornstein-Uhlenbeck process, with a free set of parameters, namely, the time-varying speed of mean reversion. We look for the speed of mean reversion that minimizes the variance of the Radon-Nikodym derivative of the target measure with respect to the initial measure under a constraint on the time integral of the variance of the state variable in the target measure.

We studied this problem in an earlier article (see Schellhorn [10]), where we explained one application of this result to the field of Monte Carlo simulation. It is sometimes important to resimulate a system under a different measure than the initial measure. The immediate example is sensitivity analysis. Another example is in the field of finance, where practitioners are often interested in seeing the results of their simulations in two different 
measures, the "actual measure," and the "risk-neutral" measure. One of these measures has typically a free parameter, or sets of parameters. Suppose the goal is to calculate $E[z]$ under two different measures, and that the integrand $z(\omega)$ - which is expensive to compute-was initially simulated in the initial measure. We argued that a computationally better resimulation estimator (compared to resimulating $z(\omega)$ in the target measure) was the sum of the initial $z(\omega)$ weighted by the Radon-Nikodym derivative $g(\omega)$ of the target measure with respect to the initial measure. However, the product $g(\omega) z(\omega)$ tends to have a larger variance than $z(\omega)$, and this fact may outweigh the performance gain of not resimulating $z$. Care must be therefore taken in selecting a target measure for simulation performance, and we suggested that a good performance measure was the variance of $g$. When the state variable $x$ is assumed Gaussian in both measures (which is very often the case in practice for better analytical tractability), the only free parameter is the speed of mean reversion $a$ of $x$ in the target measure.

The problem above is completely characterized once one of several constraints on the autocovariance function of $x$ in the target measure are introduced-we do not consider the usually less interesting case, where $x$ is not first-moment stationary in the target measure. In Schellhorn [10] we considered in turn two possible constraints:

(i) a constraint on the terminal variance of $x$,

(ii) a constraint on the average variance of $x$,

and showed that, in both cases, the control satisfied (together with other variables) a system of four nonlinear ordinary differential equations. This system happened to be quite difficult to solve numerically. Nevertheless, the so-called "change of measure" resimulation technique proved out to be effective on various examples.

Another potential application of this problem is the theory of incomplete markets in mathematical finance. Several authors (see, e.g., Rouge and El Karoui [8], Delbaen et al.[2]) explore the duality between utility maximization and optimal choice of measure. If the utility function is exponential, the dual objective to minimize is the relative entropy of the target measure, that is, the first moment of $g \log g$. If the utility function is quadratic, the dual objective to minimize is the second moment of $g$ (see Duffie and Richardson [3], Schweizer [11], Bellini and Frittelli [1]). A majority of authors seems to have pursued the first avenue, that is, minimizing entropy, because among others of its better tractability (Rheinlaender [7]). We conjecture that the result of this paper may help research in the second avenue, that is, quadratic utility functions.

In this article, we consider only a constraint on the average variance of $x$. Compared to our earlier article, we use a different representation of the second moment of $g$, which turns out to be easier to handle analytically. Using the maximum principle, we show that the optimal speed of mean reversion follows a Riccati equation. We show solutions of the problem in two cases, when volatility is constant, and when volatility is an exponential function of time. We suspect that other cases are also amenable to closed form formulae. Finally, we compare our exact results to the approximation given in Schellhorn [10].

\section{Model and results}

Notation 1. The complete filtered probability space $\left(\Omega, \mathscr{F}, P^{I}\right)$ supports a Brownian motion $W^{I}$. We use the superscripts ${ }^{I}$ and ${ }^{T}$ to refer to the probability measure, expectation 
operator, variance (Var) operator, and Brownian motion in the initial/terminal measure. When not shown otherwise, the expectation and variance operators are taken at time zero.

The dynamics of the variable $x$ of interest are

$$
\begin{gathered}
d x(t)=\sigma(t) d W^{I}(t), \\
x(0)=0,
\end{gathered}
$$

where $\sigma>0$ is a deterministic function of time. The terminal measure $P^{T}$ supports one Brownian motion $W^{T}$, with

$$
d W^{T}(t)=d W^{I}(t)+\frac{a(t) x(t)}{\sigma(t)} d t
$$

Once the speed of mean reversion $a(t)$ is specified, $P^{T}$ becomes fully specified. We define the Radon-Nikodym derivative process:

$$
g(t) \equiv E^{I}\left[\frac{d P^{T}}{d P^{I}} \mid \mathscr{F}_{t}\right] .
$$

By Girsanov theorem,

$$
d g(t)=\frac{a(t) x(t)}{\sigma(t)} d W^{I}(t)
$$

The optimization problem is to minimize the variance of $g$ under a constraint on the average variance of the state variable in the terminal measure:

$$
\begin{gathered}
\min E^{I}\left[g^{2}(t)\right] \\
\operatorname{Var}^{T}\left[\int_{0}^{\infty} x^{2}(t) d t\right] \leq A .
\end{gathered}
$$

THeOREM 2.1. The speed of mean reversion that solves (2.5) and (2.6) is of the form

$$
a(t)=\sigma^{2}(t) y(t)
$$

where $y$ solves the Riccati equation

$$
\begin{gathered}
\frac{d y(t)}{d t}=-\sigma^{2}(t) y^{2}(t)-\lambda, \\
y(T)=0,
\end{gathered}
$$

and $\lambda \geq 0$ is the Lagrange multiplier of relation (2.6).

We now look at the solution of the Riccati equation for particular volatility functions. 
4 Formulae for change of Gaussian measures

Case 1 ( $\sigma$ is constant). The solution to the Riccati equation is

$$
a(t)=\sigma \sqrt{\lambda} \tan (\sigma \sqrt{\lambda}(T-t))
$$

As required by the transversality conditions, $a(T)=0$. As expected the speed of mean reversion is increasing in $\lambda$ and decreasing in $t$. We notice that when $\lambda$ is small the speed of mean reversion is a linear decreasing function.

Case $2\left(\sigma^{2}(t)=\alpha \exp (-k t)\right.$ for $\left.\alpha>0\right)$. We write $J_{1}$ for the Bessel functions of the first kind. Let

$$
C \equiv \frac{-(1 / 2) J_{1}((2 / k) \sqrt{\lambda \alpha \exp (-k T)})-(2 / k) \sqrt{\lambda \alpha \exp (-k T)} J_{1}^{\prime}((2 / k) \sqrt{\lambda \alpha \exp (-k T)})}{J_{-1}((2 / k) \sqrt{\lambda \alpha \exp (-k T)})+(2 / k) \sqrt{\lambda \alpha \exp (-k T)} J_{-1}^{\prime}((2 / k) \sqrt{\lambda \alpha \exp (-k T)})} .
$$

Then

$$
\begin{gathered}
y(t)=-\sigma^{2}(t) \frac{k \exp (k T)}{\alpha} \frac{u^{\prime}\left(e^{k(T-t)}\right)}{u\left(e^{k(T-t)}\right)}, \\
u(s)=s^{1 / 2} J_{1}\left(\frac{2}{k} \sqrt{\lambda \alpha \exp (-k T) s}\right)+C J_{-1}\left(\frac{2}{k} \sqrt{\lambda \alpha \exp (-k T) s}\right) .
\end{gathered}
$$

Lemma 2.2. Let $v(t)=E^{T}\left[x^{2}(t)\right]$. Then

$$
E^{I}\left[g^{2}(T)\right]=\exp \left(\int_{t=0}^{T} \frac{a^{2}(t)}{\sigma^{2}(t)} v(t) d t\right)
$$

Proof. Let $\mu=a x / \sigma$. Then

$$
\begin{aligned}
E^{I}\left[g^{2}(T)\right] & =E^{T}[g(T)] \\
& =E^{T}\left[\exp \left(-\int_{0}^{T} \mu(t) d W^{I}(t)-\frac{1}{2} \int_{0}^{T} \mu^{2}(t) d t\right)\right] \\
& =E^{T}\left[\exp \left(-\int_{0}^{T} \mu(t)\left(d W^{T}(t)-\mu(t) d t\right)-\frac{1}{2} \int_{0}^{T} \mu^{2}(t) d t\right)\right] \\
& =E^{T}\left[\exp \left(\int_{0}^{T} \mu^{2}(t) d t\right)\right] .
\end{aligned}
$$


We obtain then

$$
\begin{aligned}
E^{I}\left[g^{2}(T)\right] & =E^{T}\left[\exp \left[\int_{0}^{T} \frac{a^{2}(t)}{\sigma^{2}(t)}\left[W^{T}(v(t))\right]^{2} d t\right]\right] \\
& =E^{T}\left[\exp \left[\int_{0}^{v(T)} \frac{d t}{d v} \mid \frac{a^{2}\left(v^{-1}(u)\right)}{\sigma^{2}\left(v^{-1}(u)\right)} W^{2}(u) d u\right]\right] \\
& =E^{T}\left[\exp \left[\int_{0}^{v(T)} h(u) W^{2}(u) d u\right],\right.
\end{aligned}
$$

where we have defined

$$
h(u)=\left.\frac{d t}{d v}\right|_{u} \frac{a^{2}\left(v^{-1}(u)\right)}{\sigma^{2}\left(v^{-1}(u)\right)} .
$$

To calculate the Carleman-Fredholm determinant (see, e.g., Grasselli and Hurd [4] or Levendorskii [5]), we resort to a discrete approximation. We first define $V$ as the smallest value larger than or equal to $v(T)$ so that $V / \Delta u$ is integer. We also define

$$
\begin{gathered}
H(u)=\int_{u}^{v(T)} h(s) d s \\
\Sigma^{-1}=\left[\begin{array}{rrrr}
z(1) & \cdots & z\left(\frac{V}{\Delta u}\right)
\end{array}\right], \\
{\left[\begin{array}{rrrr}
1-2 H(\Delta u) \Delta u & -4 H(2 \Delta u) \Delta u & \vdots & -4 H(V) \Delta u \\
0 & 1-2 H(2 \Delta u) \Delta u & \vdots & \cdots \\
\vdots & \vdots & \vdots & -4 H(V) \Delta u \\
0 & \vdots & 0 & 1-2 H(V) \Delta u
\end{array}\right] .}
\end{gathered}
$$

We calculate

$$
\begin{aligned}
E^{I}\left[g^{2}(T)\right] & =E^{T}\left[\exp \left[\int_{u=0}^{v(T)} h(u) W^{2}(u)\right]\right] \\
& =\lim _{\Delta u \rightarrow 0} E^{T}\left[\exp \left[\sum_{u=1}^{V / \Delta u} h(u \Delta u) \sum_{s=1}^{u} \sum_{t=1}^{u} z(s) z(t)(\Delta u)^{2}\right]\right] \\
& =\lim _{\Delta u \rightarrow 0} \frac{1}{\sqrt{(2 \pi)^{V / 2 \Delta u}}} \int \cdots \int \exp \left(-\frac{1}{2} z \Sigma^{-1} z\right) d z
\end{aligned}
$$


6 Formulae for change of Gaussian measures

$$
\begin{aligned}
& =\lim _{\Delta u \rightarrow 0} \frac{1}{\sqrt{(1-2 H(\Delta u) \Delta) \cdots(1-2 H(V) \Delta u)}} \\
& =\lim _{\Delta u \rightarrow 0} \exp \left(\int_{0}^{v(t)} H(u) d u\right) \\
& =\exp \left(\left.\int_{u=0}^{v(T)} \int_{s=u}^{v(t)} \frac{d t}{d v}\right|_{s} \frac{a^{2}\left(v^{-1}(s)\right)}{\sigma^{2}\left(v^{-1}(s)\right)} d s d u\right) \\
& =\exp \left(\left.\int_{s=0}^{v(T)} \frac{d t}{d v}\right|_{s} \frac{a^{2}\left(v^{-1}(s)\right)}{\sigma^{2}\left(v^{-1}(s)\right)} v\left(v^{-1}(s)\right) d s\right) \\
& =\exp \left(\int_{t=0}^{T} \frac{a^{2}(t)}{\sigma^{2}(t)} v(t) d t\right) .
\end{aligned}
$$

Proof of Theorem 2.1. The problem is

$$
\begin{gathered}
\min _{a} \int_{0}^{T}\left(\frac{a^{2}(t)}{\sigma^{2}(t)}+\lambda\right) v(t) d t \\
\frac{d v(t)}{d t}=-2 a(t) v(t)+\sigma^{2}(t), \\
v(0)=0 .
\end{gathered}
$$

The Hamiltonian is

$$
H(v(t), a(t), t)=-\left(\frac{a^{2}(t)}{\sigma^{2}(t)}+\lambda\right) v(t)+z(t)\left(-2 a(t) v(t)+\sigma^{2}(t)\right)
$$

The Pontryagin optimality conditions are

$$
\begin{gathered}
\frac{\partial H}{\partial a}=-\frac{2 a v}{\sigma^{2}}-2 z v=0, \\
\frac{d z(t)}{d t}=\frac{a^{2}(t)}{\sigma^{2}(t)}+\lambda+2 a(t) z(t), \\
z(T) v(T)=0 .
\end{gathered}
$$

We note that these optimality conditions are sufficient (see Mangasarian [6]). From (2.20) we obtain

$$
z(t)=-\frac{a(t)}{\sigma^{2}(t)}
$$


which we reinsert in $(2.21)$

$$
\frac{d}{d t}\left(\frac{a(t)}{\sigma^{2}(t)}\right)=-\frac{a^{2}(t)}{\sigma^{2}(t)}-\lambda .
$$

We let $y=a / \sigma^{2}$ and obtain the result. The transversality condition (2.22) imposes $a(T)=0$.

\section{Example}

In this section, we compare on two examples the optimal control given by the solution of the theorem, to the approximate optimal control given in Schellhorn [10]. We now expose briefly the approximation approach. In the latter article, we did not exploit the lemma, but used the following representation for our objective:

$$
E^{I}\left[g^{2}(T)\right]=\exp \left[\int_{0}^{T} \sigma^{2}(t) f(t) d t\right]
$$

where

$$
\begin{gathered}
\frac{d f(t)}{d t}=-\frac{a^{2}(t)}{\sigma^{2}(t)}+4 a(t) f(t)-2 \sigma^{2}(t) f^{2}(t), \\
f(T)=0 .
\end{gathered}
$$

The representation (3.1)-(3.2) when inserted in the optimization problem (2.5), (2.6) results in optimal control problem involving two state variables: $f$ and $v$. The optimality conditions of that problem (which were not even sufficient) turned out to be quite difficult to solve numerically. Instead, we suggested to reduce the state space to only one variable, in a line similar to Sannutti [9].

The approximated optimal control follows, then

$$
a_{\text {approx }}(t)=-\frac{\sigma^{2}(t) z(t) v(t)}{\int_{0}^{t} \sigma^{2}(u) d u},
$$

where the costate variable $z$ follows:

$$
\frac{d z}{d t}=\lambda+2 a z
$$

under the terminal constraint $z(T)=0$.

We report in Figures 3.1 and 3.2 our results for two different volatilities:

(i) $\sigma(t)=0.2$ in Figure 3.1;

(ii) $\sigma(t)=0.2(1+0.2 \cos (t / 4))$ in Figure 3.2.

In both cases, the relative average variance of $x$ is the ratio between the cumulated variance $\operatorname{Var}^{T}\left[\int_{0}^{T} x^{2}(t) d t\right]$ "with mean reversion" and the cumulated variance $\operatorname{Var}^{I}\left[\int_{0}^{T} x^{2}(t) d t\right]$ "without mean reversion." Since the constraint (2.6) is clearly tight, the numerator of this ratio is equal to our constraint $A$. On the $y$-axis, we report the logarithm of the second moment of $g(T)$, calculated according to the representation of this article, that is, (2.12). 
8 Formulae for change of Gaussian measures

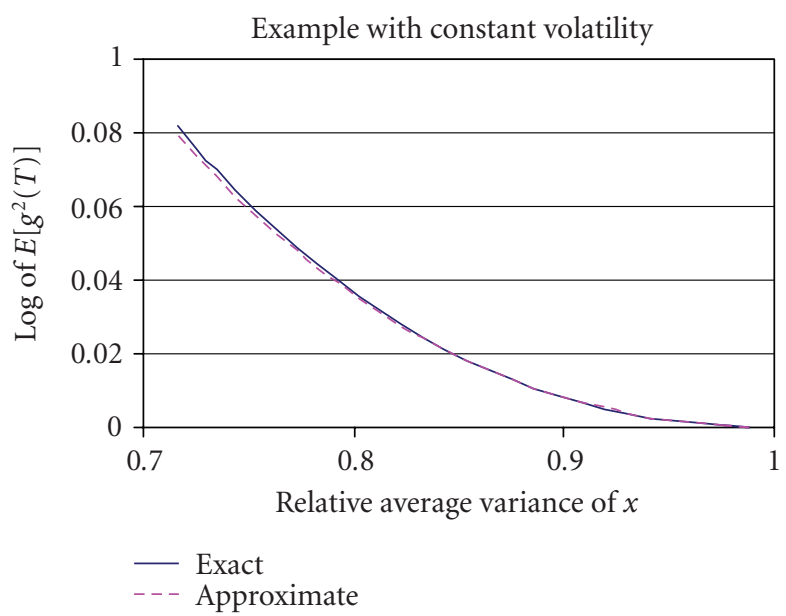

Figure 3.1. Logarithm of $E\left[g^{2}(T)\right]$ as a function of the ratio of $A$ over the cumulated variance of $x$ in the uncontrolled case $(a=0)$. The volatility is $\sigma(t)=0.2$ and $T=3$.

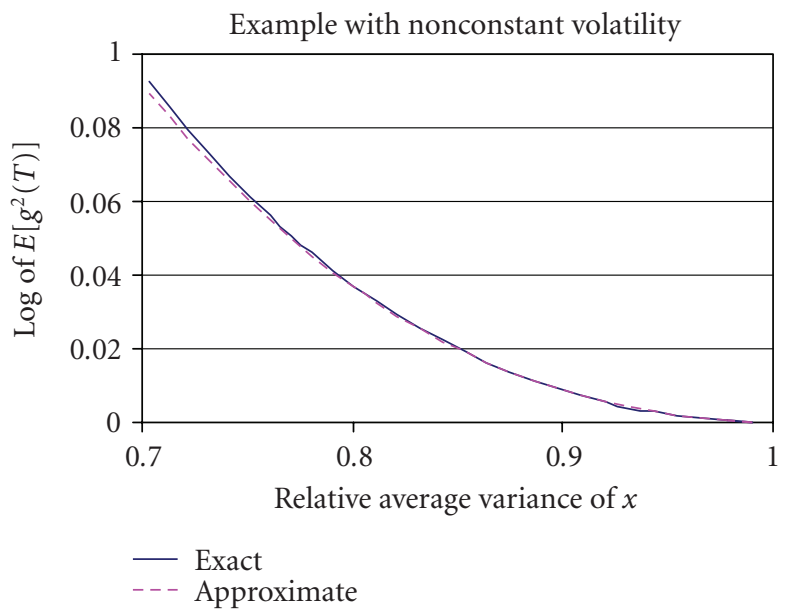

Figure 3.2. Logarithm of $E\left[g^{2}(T)\right]$ as a function of the ratio of $A$ over the cumulated variance of $x$ in the uncontrolled case $(a=0)$. The volatility is $\sigma(t)=0.2(1+0.2 \cos ((t / 4)))$ and $T=3$.

It turns out that both methods, the exact method of this article, and the approximate one, yield remarkably similar results in these two examples.

\section{Conclusions}

This article provides an alternate characterization of the solution of an optimal control problem first introduced in the literature by us in Schellhorn [10]. The result presented in this article is stronger, since it is not the result of a reduction of the problem. The 
examples we presented show however a remarkable coincidence in results between both methods. We emphasize that this needs not be the case.

Our methodology can be applied to Monte Carlo resimulation, that is, simulation in two different measures. We reported in our earlier article that the "change of measure" resimulation scheme, where we simulate the cash flows $z(\omega)$ only once (to calculate market value), and then adjust them by $g(\omega)$ to calculate the empirical distribution, was up to twice faster than a "traditional scheme," where two independent simulations were performed. The same speed improvement can be attained using the method presented here.

\section{References}

[1] F. Bellini and M. Frittelli, On the existence of minimax martingale measures, Mathematical Finance 12 (2002), no. 1, 1-21.

[2] F. Delbaen, P. Grandits, T. Rheinländer, D. Samperi, M. Schweizer, and C. Stricker, Exponential hedging and entropic penalties, Mathematical Finance 12 (2002), no. 2, 99-123.

[3] D. Duffie and H. R. Richardson, Mean-variance hedging in continuous time, The Annals of Applied Probability 1 (1991), no. 1, 1-15.

[4] M. R. Grasselli and T. R. Hurd, Wiener chaos and the Cox-Ingersoll-Ross model, Proceedings of The Royal Society of London. Series A. Mathematical, Physical and Engineering Sciences 461 (2005), no. 2054, 459-479.

[5] S. Levendorskii, Pseudo-diffusions and quadratic term structure models, Working paper, University of Texas, Austin, January 2004.

[6] O. L. Mangasarian, Sufficient conditions for the optimal control of nonlinear systems, SIAM Journal on Control and Optimization 4 (1966), no. 1, 139-152.

[7] T. Rheinlaender, private communication, 2004.

[8] R. Rouge and N. El Karoui, Pricing via utility maximization and entropy, Mathematical Finance 10 (2000), no. 2, 259-276.

[9] P. Sannutti, Singular perturbation method in the theory of optimal control, Report R-379, University of Illinois, Illinois, 1966.

[10] H. Schellhorn, Optimal changes of Gaussian measures, with an application to finance, Under second round of revision with Applied Mathematical Finance, 2006.

[11] M. Schweizer, Approximation pricing and the variance-optimal martingale measure, The Annals of Probability 24 (1996), no. 1, 206-236.

Henry Schellhorn: School of Mathematical Sciences, Claremont Graduate University, Claremont, CA 91711, USA

E-mail address: henry.schellhorn@cgu.edu 


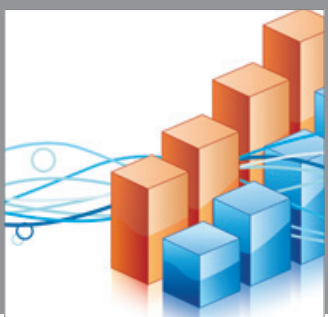

Advances in

Operations Research

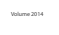

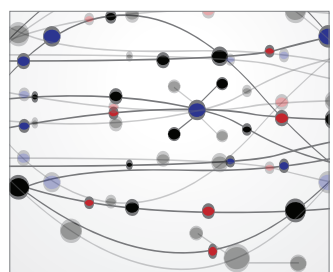

\section{The Scientific} World Journal
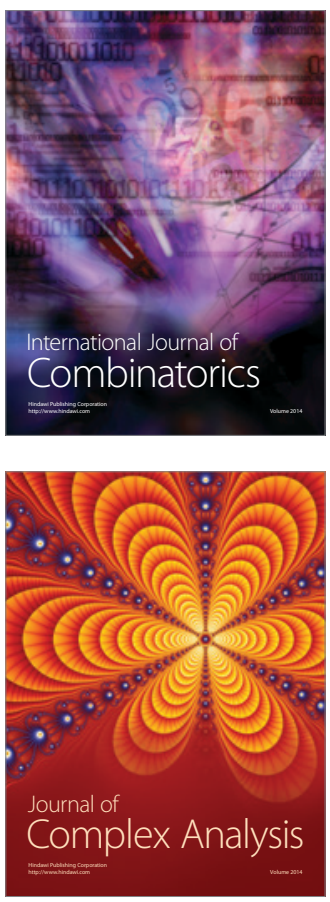

International Journal of

Mathematics and

Mathematical

Sciences
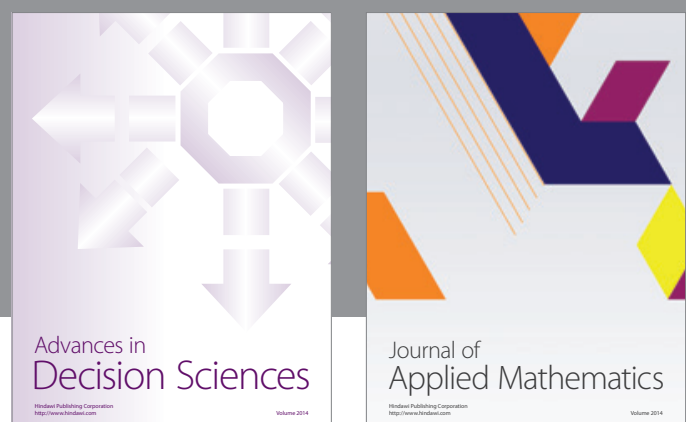

Journal of

Applied Mathematics
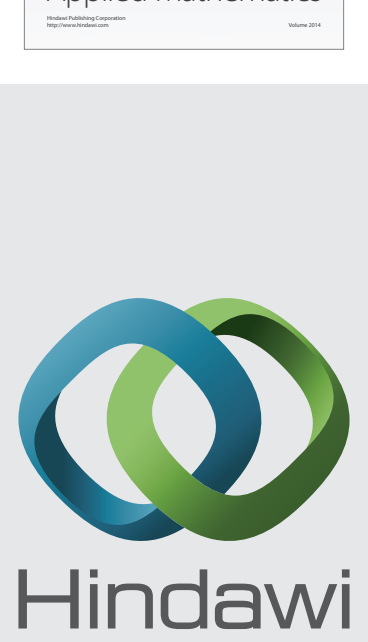

Submit your manuscripts at http://www.hindawi.com
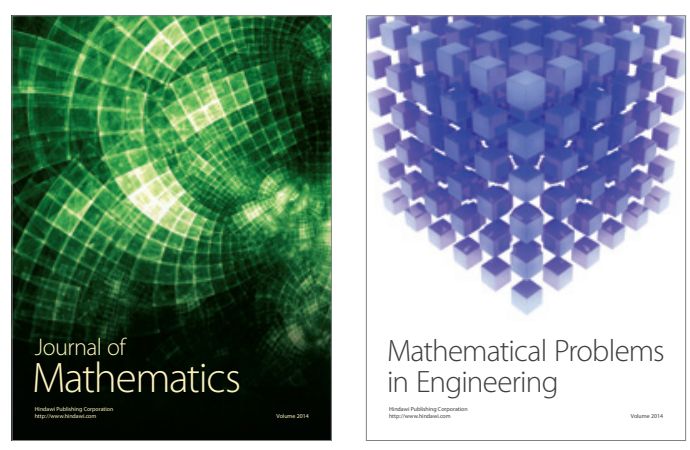

Mathematical Problems in Engineering
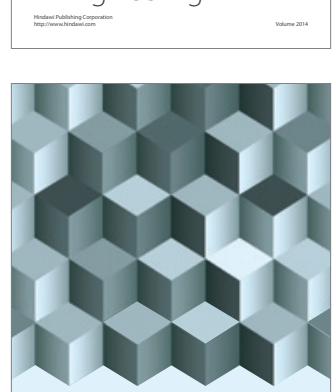

Journal of

Function Spaces
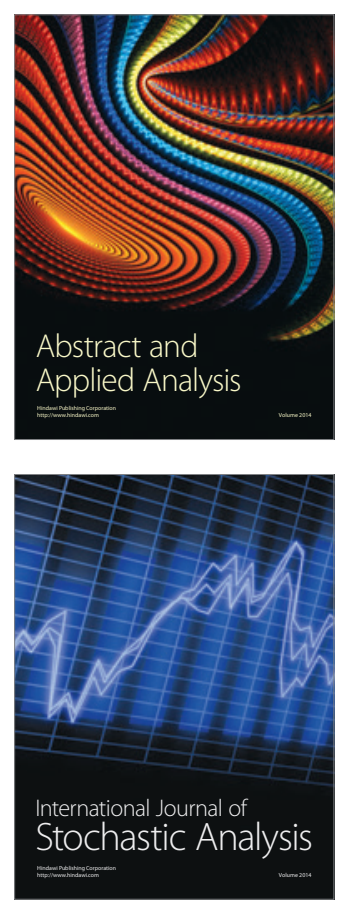

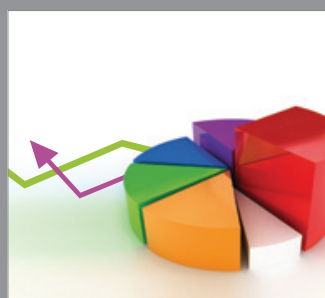

ournal of

Probability and Statistics

Promensencen
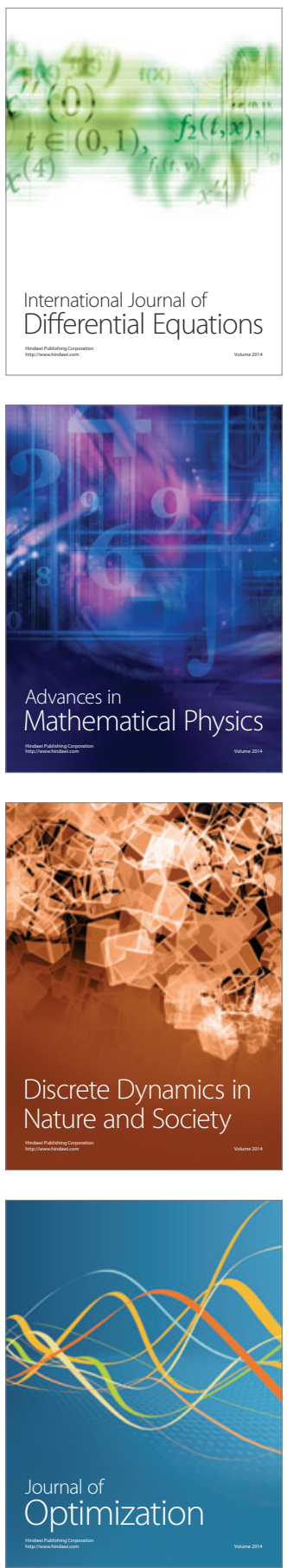